\title{
ANALYSIS OF E-PLANE STRUCTURES BY THE COUPLED-INTEGRAL-EQUATIONS TECHNIQUE
}

\author{
${ }^{1}$ Smain Amari, ${ }^{1}$ Jens Bornemann* and ${ }^{2}$ Rüdiger Vahldieck \\ ${ }^{1}$ University of Victoria, Victoria, B.C., Canada \\ ${ }^{2}$ Swiss Federal Institute of Technology, Zürich, Switzerland
}

\section{INTRODUCTION}

Waveguide discontinuities are essential elements in modern microwave frequency selective devices such as filters, matching networks and polarizers [1]. The performance of these microwave components is contingent upon an accurate design and analysis of the interactions between the different discontinuities in the waveguide.

The regular shape of commonly encountered discontinuities in waveguide components makes their analysis by the Mode-Matching Technique (MMT) exceedingly comvenient [2]. In this approach, the generalized scattering matrix of the structure is determined by cascading the scattering matrices of the different discontinuities [1]. 'I'he method is, however, known to converge slowly when sharp metallic edges are present in the structure as the modes of the uniform sections fail to include the singular nature of the electromagnetic field.

A modified version of the Mode-Matching Technique consists in including the edge condition to compute the scattering matrices of the individual discontinuities and then cascading the thus obtained scattering matrices to obtain the overall response of the structure [3]. The size of the individual scattering matrices is reduced by using the fact that in most sections only a few modes are propagating or active [3].

In this work, we propose to modify the Mode-Matching Technique much further by essentially stripping the normal modes of the individual section of any essential role in computing the interactions between the different discontinuities. The mumerical efficiency achieved in analyzing a single discontinuity by performing a change of basis at the discontinuity is extended to an arbitrary number of interacting discontinuities. To achieve this result, we perform changes of basis at each of the discontinuities simultaneously, thereby reformulating the original problem in terms of a set of coupled integral equations. By directly concentrating on the discontinuities themselves, which are responsible for the physics of the device, the modes of the uniform sections play only a secondary role, mainly in computing imer products. In addition, in most of these devices, the engineer is only interested in the reflection and transmission of the fundamental mode at the input and output. 'The CIE'I' allows us to "directly" and accurately compute these transfer functions.

Numerical results obtained from the CIE'T are compared to data from the literature and measurements to demonstrate the accuracy and speed of the techrique. 


\section{THEORY}

The Coupled-Integral-Equations Technique (CIET) is described in details in [4]; here we only sketch the essential points.

Consider the structure shown in Figure 1a. It consists of a single E-plane stub in a rectangular waveguide. It is assumed that all metallic walls are lossless and that only the fundamental mode $T E_{10}$ is incident, with amplitude equal to unity, from the left-side of the structure.

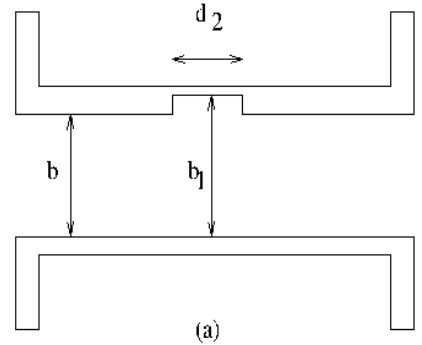

(a)

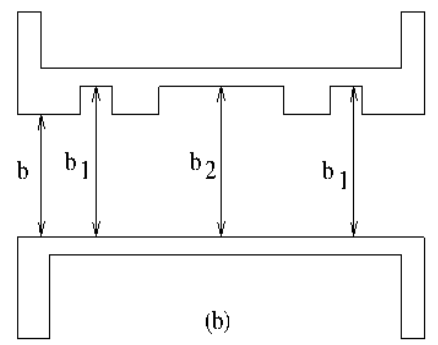

(b)

Figure 1: A single E-plane stub (a) and three E-plane stubs (b).

Following the MMT, the transverse components of the electromagnetic field are expanded in modal series in each region. In the left-most region, reflected waves in addition to the incident fundamental mode are present. On the other hand, waves traveling in both directions are present in the internal region. Finally, there are only transmitted waves in the right-most region. Two coupled integral equations for the transverse electric fields $X_{1}$ and $X_{2}$ at the two discontinuities are derived from the boundary conditions of $E_{y}$ and $H_{x}$. More explicitly, these are written as

$$
\begin{array}{r}
\sum_{m=1}^{\infty} Y_{m}^{I I} \frac{\tilde{X}_{1}^{I I}(m) \cos \left[k_{m b}^{I I} d\right]-\tilde{X}_{2}^{I I}(m)}{j \sin \left[k_{m}^{I I} d_{2}\right]} \cos \left[(m-1) \pi \frac{y}{b_{1}}\right] \\
+\sum_{m=1}^{\infty} Y_{m}^{I} \tilde{X}_{1}^{I}(m) \cos \left[(m-1) \pi \frac{y}{b}\right]=2 Y_{1}^{I} \\
\sum_{m=1}^{\infty} Y_{m}^{I I} \frac{\tilde{X}_{2}^{I I}(m) \cos \left[k_{m}^{I I} d_{2}\right]-\tilde{X}_{1}^{I I}(m)}{j \sin \left[k_{m}^{I I} d_{2}\right]} \cos \left[(m-1) \pi \frac{y}{b_{1}}\right] \\
+\sum_{m=1}^{\infty} Y_{m}^{I} \tilde{X}_{2}^{I}(m) \cos \left[(m-1) \pi \frac{y}{b}\right]=0
\end{array}
$$

Here, $Y_{m}^{i}$ are the wave admittances of the $T E_{1 m}^{x}$ modes, and the following notation was introduced

$$
\tilde{X}_{i}^{I}(m)=\frac{2}{b\left(1+\delta_{m \mathbf{1}}\right)} \int_{0}^{b} X_{i}(y) \cos \left[(m-1) \pi \frac{y}{b}\right] d y
$$




$$
\tilde{X}_{i}^{I I}(m)=\frac{2}{b_{1}\left(1+\delta_{m 1}\right)} \int_{0}^{b} X_{i}(y) \cos \left[(m-1) \pi \frac{y}{b_{1}}\right] d y
$$

To solve this set of coupled integral equations, we expand the functions $X_{i}(y)$ in series of basis functions which include the edge conditions and apply Galerkin's method to obtain a set of linear equations in the expansion coefficients. Once these are determined the reflection and transmission properties follow straightforwardly.

'l'he case where more than two discontinuities are present (Figure 1b) is analyzed similarly.

\section{RESULTS}

The CIET and the MMT are applied to the analysis of a single E-plane stub as well as a three $\mathrm{E}$-plane stubs (Figure $1 \mathrm{a}$ and $1 \mathrm{~b}$ ).

Figure 2 shows the reflection coefficient $S_{11}$ as a function of frequency as obtained from the CIET using 2 basis functions and the MMT using 5 modes. The stars are experimental values from reference [3]. Note that the CIFT results fall closer to the experimental values whereas those from MMT are practically identical to the simulated data in [3] where the edge condition is included in a modular fashion. More basis functions where used in the CIE' without any noticeable effect.

Figure 3 shows the reflection coefficient versus frequency of the three-stub structure in figure $1 \mathrm{~b}$. The dimensions are those given in [3]. The CIFT comverges with 3 basis functions. The dashed line are the results from the MMT with 15 modes and the dotted line with 5. Again the stars are experimental results from [3]. Note that the CIET predicts more accurately the experimental results or those form the MM'T with a 15 modes. 'T'he results from the modified MMT as presented in [3] coincide with those of the standard MMT with 5 modes. This is not surprising as the overall response of the structure is obtained from cascading the individual scattering matrices which directly involve the modes of the uniform sections.

\section{CONCLUSION}

The Coupled-Integral-Equations Technique is successfully applied to accurately analyze E-plane structures in one step. A considerable reduction in the numerical effort is achieved along with an increase in accuracy by including the edge conditions at all the discontinuities. Excellent agreement with measured data is demonstrated with only two to three basis functions per discontinuity.

\section{REFERENCES}

1. J. Uher, J. Bornemann and Uwe Rosenberg, Waveguide Components for Antenna Feed Systems: Theory and CAD, Artech House, Boston 1993.

2. T. Itoh ed., Numerical Techniques for Microwave and Millimeter-Wave Passive Structures, Johrı Wiley \& Sons, New York 1989. 
3. T. Rozzi and M. Mangiardo, "E-plane steps in rectangular waveguide," IEEE Trans. Microwave Theory Tech., vol. 39, pp. 1279-1288, August 1991. 4. S. Amari, J. Bornemamn and R. Vahldieck, "Accurate analysis of scattering from multiple waveguide discontinuities using the coupled-integral-equations technique," J. Electromagnetic Waves and Applications, Vol. 10, No. 12, pp. 1623-1644, 1996.

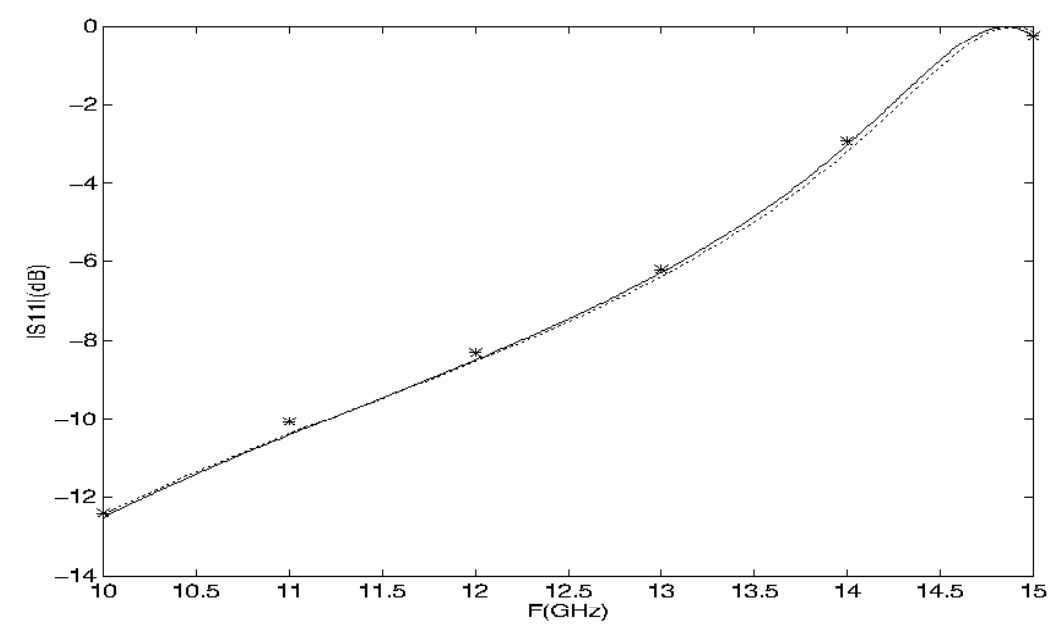

Figure 2: $S_{11}(\mathrm{~dB})$ of Figure 1a as a function of frequency as obtained from the CIET with 2 basis functions (solid line) and the MMT with 5 modes (dashed line). The stars are measurement from [3]

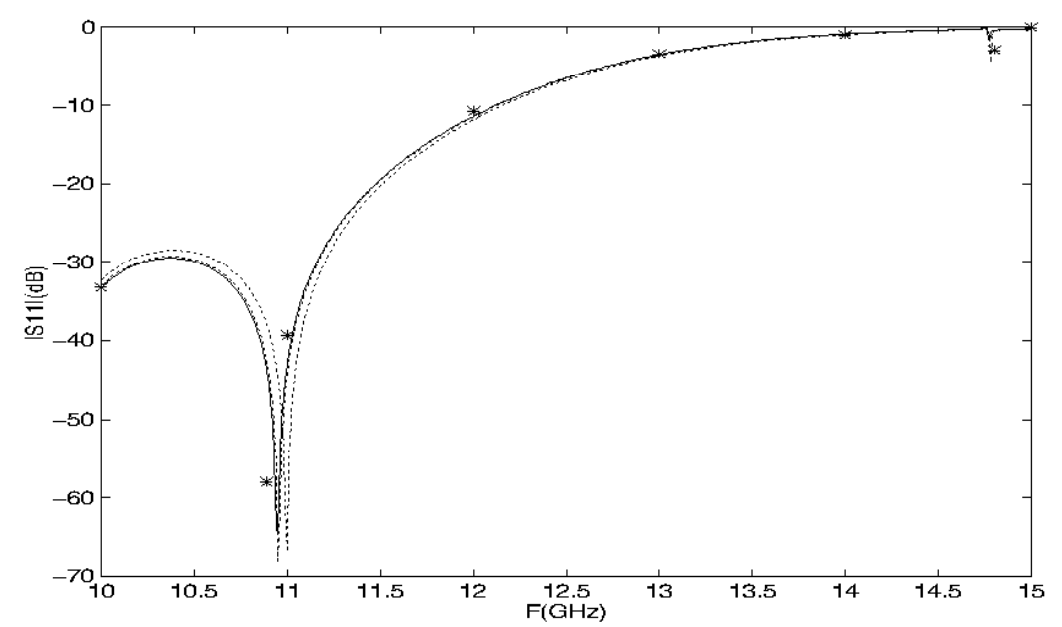

Figure 3: $S_{11}(\mathrm{~dB})$ of Figure $1 \mathrm{~b}$ as a function of frequency as obtained from the CIET (solid line) with 4 basis functions and the MMT with 5 (dotted line) and 15 (dashed line) modes. T'he stars are measurement from [3]. 